\title{
Qualidade das prescrições de profilaxia para tromboembolismo venoso em um hospital universitário no Brasil
}

\author{
Quality of venous thromboembolism prophylaxys in a university hospital in Brazil
}

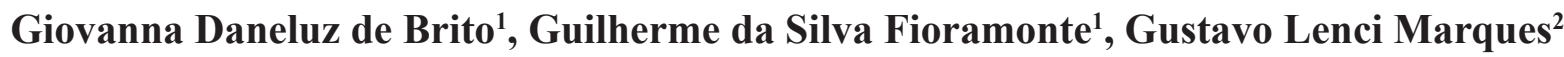

Brito GD, Fioramonte GS, Marques GL. Qualidade das prescrições de profilaxia para tromboembolismo venoso em um hospital universitário no Brasil / Quality of venous thromboembolism prophylaxys in a university hospital in Brazil. Rev Med (São Paulo). 2020 jan.-fev.;99(1):1-7.

RESUMO: Objetivo: Embora preconizada, a profilaxia para tromboembolismo venoso não é sempre corretamente prescrita. O objetivo deste estudo é avaliar o nível de adequação das prescrições de profilaxia para tromboembolismo venoso para pacientes internados em um hospital universitário. Métodos: Trata-se de um estudo analítico transversal no qual foram avaliadas prescrições para pacientes internados nas unidades clínicas, cirúrgicas e críticas de um hospital universitário durante 30 dias. Excluíram-se pacientes em uso de anticoagulantes para finalidade diferente da profilática. Os critérios de avaliação da profilaxia de tromboembolismo venoso basearam-se na $9^{\text {a }}$ Diretriz do American College of Chest Physicians. Resultados: 358 prescrições foram analisadas. 65 foram excluídas. Das 293 prescrições selecionadas, $106(36,18 \%)$ foram consideradas incorretas. A subprescrição foi a principal causa de inadequação, observada em 72 casos $(67,9 \%)$. Inadequações foram maiores nas unidades cirúrgicas $(48,72 \%)$ do que nas clínicas $(35,11 \%)$ e críticas $(26,2 \%)(p=0,0013)$. Conclusões: Preocupantes taxas de inadequação da profilaxia para tromboembolismo venoso para pacientes internados foram encontradas, havendo predomínio da subprescrição. As maiores inadequações foram encontradas nas unidades cirúrgicas. Intervenções são necessárias para otimizar essa importante prática de segurança ao paciente hospitalizado.

Descritores: Trombose/prevenção \& controle; Tromboembolia venosa/prevenção \& controle; Prevenção de doenças; Hospitalização; Prescrições; Anticoagulantes; Embolia pulmonar, Heparina.

\begin{abstract}
Objective: Though recommended, prophylaxis for venous thromboembolism is not always correctly prescribed. In light of this fact, our objective is to evaluate the quality of medical prescriptions with regards to venous thromboembolism prophylaxis in a brazilian teaching hospital. Methods: During a 30-day period, medical prescriptions to patients treated at the internal medicine ward, surgery ward and at the intensive care unit were assessed. Patients on anticoagulants for a non-prophylactic reason were excluded. This cross-sectional analysis evaluated the appropriateness of venous thromboembolism prophylaxis based on the 9th Guideline of the American College of Chest Physicians. Results: 358 prescriptions have been firstly assessed, of which 65 were excluded. Out of 293 selected prescriptions, $106(36.2 \%)$ were considered inaccurate. The main cause of inadequacy was underprescription, which was observed in 72 cases $(67.9 \%)$. Surgery wards accounted for the majority of inaccurate precriptions $(48.72 \%)$ compared to internal medicine $(35.11 \%)$ and critical care ones $(26.2 \%)(\mathrm{p}=0.0013)$. Conclusion: Alarming rates of inadequate prescriptions regarding venous thromboembolism prophylaxis were found. Underprescription was the main cause of inadequacy. Surgery wards were responsible for most of the imprecision. Further interventions are required in order to improve this important and safe medical practice for hospitalized patients.
\end{abstract}

Keywords: Thrombosis/prevention \& control; Venous thromboembolism/prevention \& control; Disease prevention; Hospitalization; Prescriptions; Anticoagulants; Pulmonary embolism; Heparin.

\footnotetext{
1. Acadêmicos do Curso de Medicina da Universidade Federal do Paraná, Curitiba, Paraná, Brasil. ORCID: Brito, GD - https://orcid.org/0000-00017279-9366; Fioramonte, GS - https://orcid.org/0000-0002-8505-1258. E-mail: giovannadbrito@gmail.com, guifioramonte@gmail.com.

2. Professor adjunto do Departamento de Clínica Médica da Universidade Federal do Paraná. Médico cardiologista do Hospital de Clínicas da Universidade Federal do Paraná. Coordenador Adjunto do Curso de Medicina da Pontifícia Universidade Católica do Paraná, Curitiba, PR. ORCID: http://orcid. org/0000-0002-6057-0350. E-mail: gustavolencimarques@gmail.com.

Endereço para correspondência. Rua Engenheiro Niepce da Silva, 144, Apto 1403C, Portão. Curitiba, PR, BR. CEP: 80.610-280.
} 


\section{INTRODUÇÃO}

Ser internado em qualquer hospital do mundo Mundial da Saúde, estima-se que 1 em cada 10 pacientes sofre algum tipo de dano durante a hospitalização, sendo que metade deles poderiam ter sido evitados ${ }^{1}$. Entre os potenciais causadores de danos a pacientes internados, destaca-se o tromboembolismo venoso (TEV).

O tromboembolismo venoso é resultado de um estado de hipercoagulabilidade, e inclui a trombose venosa profunda (TVP) de membros inferiores e pelve, além de sua complicação, a embolia pulmonar (EP). Trata-se de uma doença multifatorial, originada a partir de interações entre dois principais componentes: predisposições à trombose, que podem ser herdadas ou adquiridas, e uma variedade de fatores de risco. É um agravo frequente, com incidência semelhante à de acidente vascular encefálico ${ }^{2}$. Cerca de $25 \%$ dos episódios de TEV estão associados à hospitalização ${ }^{3}$. Em média, 50-75\% dos casos ocorrem dentro dos próprios hospitais ${ }^{(4)}$ e estima-se que estão associados a $10 \%$ das mortes de pacientes internados ${ }^{5}$.

Além dos altos índices de mortalidade, a ocorrência de TEV também está relacionada ao desenvolvimento de complicações em pacientes internados, onerando os cofres hospitalares ${ }^{6}$. Em um estudo populacional, a previsão de custo médio foi 2,5 vezes maior para pacientes com TEV associada a hospitalização atual ou recente por doença aguda em comparação a pacientes controle hospitalizados com câncer ${ }^{7}$.

Esse cenário indesejável, entretanto, poderia ser evitado. Diversos estudos já demonstraram que o uso de anticoagulantes é capaz de reduzir até $63 \%$ do risco relativo de TEV durante a hospitalização $0^{8,9}$, tendo sido, por isso, considerada como sendo "o número um na prática de segurança de pacientes" em hospitais pela Agency for Healthcare Research and Quality ${ }^{10}$.

Mesmo assim, a taxa de prescrições de profilaxias em hospitais ainda estão longe de ser adequadas. Diversos estudos brasileiros e internacionais revelam que a profilaxia para TEV é, em geral, subprescrita, resultando num cenário no qual muitos pacientes internados encontram-se em risco da ocorrência de TEV. ${ }^{(11-13)} \mathrm{O}$ tromboembolismo venoso ainda é considerada a principal causa de morte evitável em hospitais ${ }^{14}$.

Por essas razões, o objetivo desse estudo é o de avaliar o nível de adequação das prescrições de profilaxias para tromboembolismo venoso para pacientes internados em um hospital de ensino.

\section{MÉTODOS}

Trata-se de um estudo analítico transversal no qual foram analisadas prescrições médicas de profilaxias para
TEV para pacientes maiores de 18 anos que estiveram internados no período de 19/07/2017 a 19/08/2017 em enfermarias clínicas e cirúrgicas, e em unidades críticas de um hospital universitário. Foram excluídas do estudo as prescrições de anticoagulantes para outras finalidades que não a profilática. Prescrições de pacientes cujas condições clínicas interferiam na indicação de profilaxias também foram excluídas (listados na seção Resultados).

Os critérios usados para avaliação da prescrição da profilaxia para TEV basearam-se na $9^{\text {a }}$ Diretriz para Profilaxia de TEV do American College of Chest Physicians $\left(9^{\mathrm{a}} \mathrm{ACCP}\right)$, que preconiza que pacientes com alto risco para o desenvolvimento de TEV devem receber anticoagulantes profiláticos. Para a estratificação de risco, o escore de Pádua foi utilizado para pacientes clínicos ${ }^{15}$, e o escore de Caprini, para os cirúrgicos ${ }^{16}$. Foram consideradas adequadas, quando indicadas, as profilaxias para TEV prescritas nas seguintes posologias:

1. Heparina de Baixo Peso Molecular (HBPM) 40 mg subcutânea (SC) uma vez ao dia, ou;

2. Heparina Não-Fracionada (HNF) 5.000 UI SC de 12 em 12 h ou de 8 em 8 h, ou;

3. Fondaparinux $2,5 \mathrm{mg}$ SC uma vez ao dia.

Para doentes renais crônicos, é preconizada a correção da posologia, tendo sido considerada adequada quando prescrita nas seguintes posologias:

1. HBPM $20 \mathrm{mg}$ ao dia, ou;

2. HNF 5.000 UI de $8 / 8 \mathrm{~h}$ ou de $12 / 12 \mathrm{~h}$.

O processo de avaliação das prescrições está ilustrado na Figura 1.

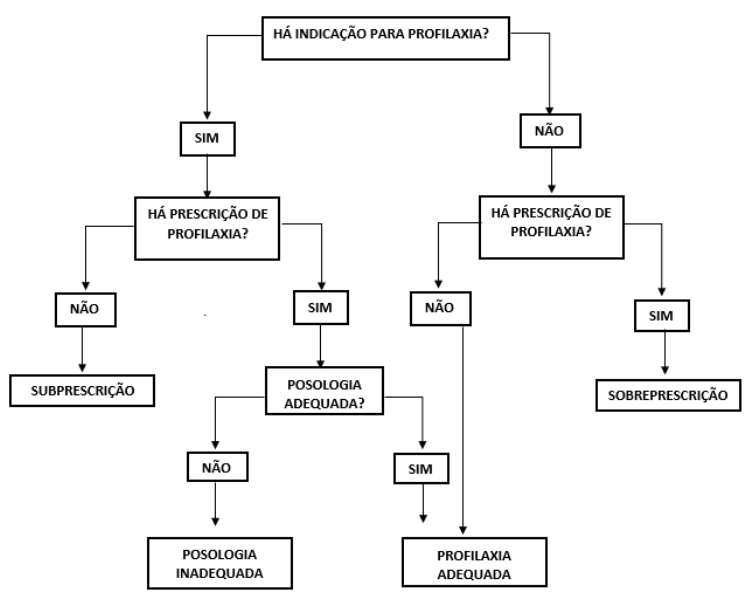

Figura 1 - Processo de avaliação das prescrições profiláticas 
O fluxograma ilustra como as prescrições para profilaxia de tromboembolismo foram avaliadas. Quando havia indicação para prescrição, mas essa não era prescrita, a categoria em que essa prescrição foi enquadrada foi a de subprescrição. Quando havia indicação, e ela era prescrita, passava-se para uma nova etapa, que era a análise da posologia. Se fosse inadequada, a categoria foi a intitulada posologia inadequada; se adequada, a precrição foi considerada profilaxia adequada. Por outro lado, quando não havia indicação para profilaxia, mas essa era prescrita, era caracterizada a sobreprescrição; quando não indicada, e não prescrita, foi considerada profilaxia adequada.

O estudo foi aprovado pelo Comitê de Ética em Pesquisa do Hospital de Clínicas da Universidade Federal do Paraná (Número do projeto: 775445 | Número do parecer: 2.131.561 | Data do parecer: 22 de junho de 2017) e, antes do início da coleta diária de dados, o médico assistente de cada paciente assinou Termo de
Consentimento Livre e Esclarecido.

Os dados coletados foram armazenados em planilha do Microsoft Excel. O nível de adequação das profilaxias foi determinado segundo a concordância entre a conduta vigente e o recomendado pelas diretrizes. Os resultados obtidos por variáveis qualitativas foram descritos por frequências e percentuais. Para inferência de associação entre duas variáveis qualitativas, foram utilizados os testes qui-quadrado e teste exato de Fischer. Significância estatística considerada foi de $5 \%$. Os dados foram analisados pelo Software R Core Team, versão 3.4.017.

\section{RESULTADOS}

No total, 358 prescrições para profilaxia de TEV foram analisadas (Tabela 1). A média de idade dos pacientes da amostra foi de 57,7 anos (18-96, DP 16,7).

Tabela 1. Distribuição dos pacientes selecionados por unidade de internamento

\begin{tabular}{lllll}
\hline & \multicolumn{3}{c}{ Unidades } & Geral \\
\cline { 2 - 4 } & Clínicas & Cirúrgicas & Críticas & \multirow{2}{*}{$358(100 \%)$} \\
\hline $\mathrm{N}^{\mathrm{o}}$ de prescrições & $170(47,5 \%)$ & $85(23,7 \%)$ & $103(28,8 \%)$ & \\
Sexo do paciente & & & & $174(48,6 \%)$ \\
Masculino & $74(43,5 \%)$ & $45(52,9 \%)$ & $55(53,4 \%)$ & $183(51,1 \%)$ \\
Feminino & $95(55,9 \%)$ & $40(47,1)$ & $48(46,6 \%)$ & $1(0,3 \%)$ \\
Mulher transgênero & $1(0,6 \%)$ & - & - & $57,7 \pm 16,7$ \\
Idade do paciente (anos) & $60,0 \pm 15,8$ & $51,3 \pm 16,6$ & $59,3 \pm 17,1$ & \\
\hline
\end{tabular}

FONTE: Os autores (2018).

Das 358 prescrições analisadas, 65 possuíam critérios para exclusão do estudo de TEV $(18,16 \%)$. Os motivos da exclusão estão listados na Figura 2. Entre os 47 pacientes sob anticoagulação plena, 19 possuíam o diagnóstico de tromboembolismo venoso.

Foram eleitas 358 prescrições foram para análise, sendo 65 delas excluídas, de modo que, ao final, 293 prescrições foram incluídas no estudo.

A Figura 3 apresenta os resultados da análise geral de profilaxias para TEV.

As prescrições consideradas inadequadas devido à posologia incorreta $(\mathrm{n}=15)$ possuíam a seguinte apresentação na análise de TEV: Enoxaparina $40 \mathrm{mg} / \mathrm{dia}$ em doente renal ( $\mathrm{n}=8)$; Enoxaparina $20 \mathrm{mg} / \mathrm{dia}$ em paciente sem doença renal $(\mathrm{n}=6)$; e Enoxaparina $40 \mathrm{mg}$ 12/12h $(\mathrm{n}=1)$. Entre as unidades analisadas, as cirúrgicas foram as que apresentaram as maiores taxas de inadequação da prescrição da profilaxia $(48,72 \%$; $\mathrm{p}=0,0013)$ (Tabela 2$)$.

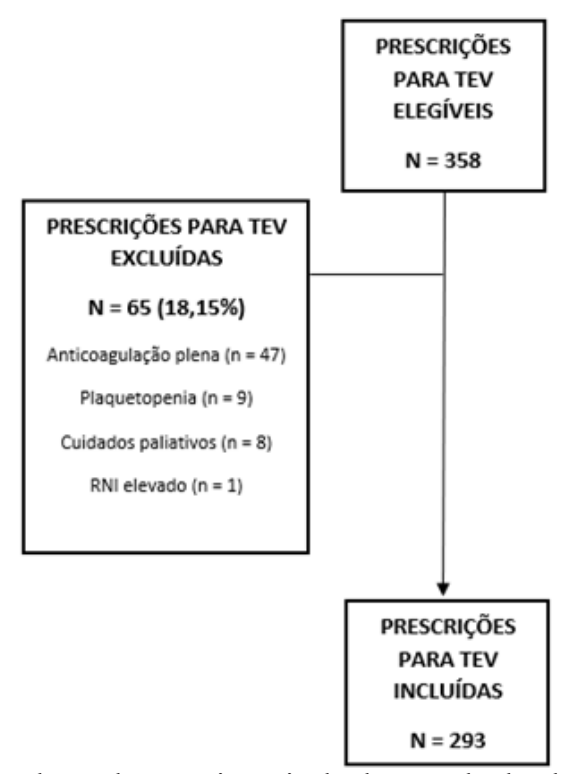

Figura 2 - Relação de prescrições incluídas e excluídas do estudo 
A

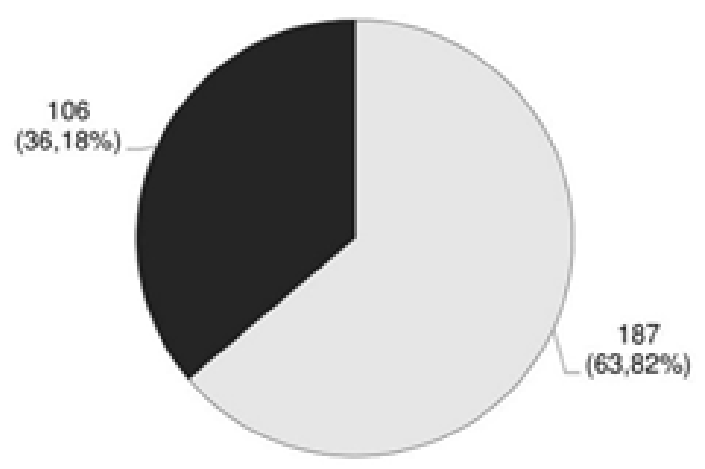

$\square$ Prescriçōes corretas

- Prescriçōes incorretas
B

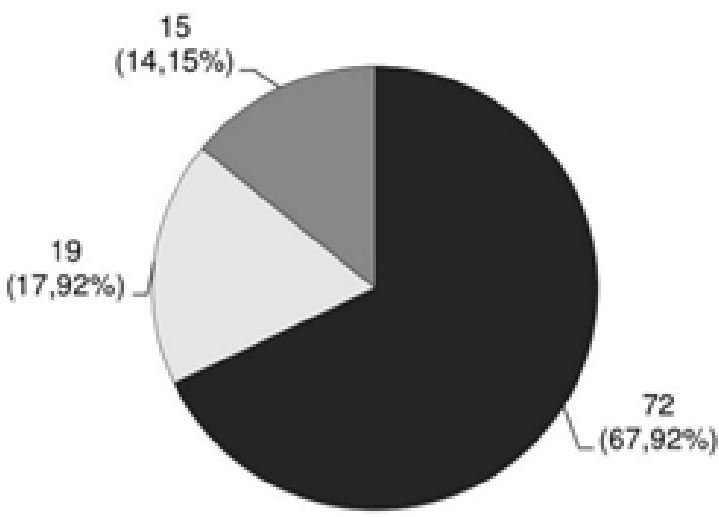

• Subprescrição $\quad \square$ Sobreprescrição a Posologia incorreta

Fonte: Autores, 2018.

Figura 3 - Análise geral de profilaxias para TEV. O gráfico A ilustra a distribuição geral dos níveis de adequação das prescrições de profilaxia para TEV, e o gráfico B relaciona a distribuição geral dos motivos de inadequação entre as prescrições incorretas para TEV

Tabela 2 - Análise da adequação de prescrições para profilaxia de TEV por unidade de internamento

\begin{tabular}{lcccc}
\hline & \multicolumn{3}{c}{ Unidades } & \\
\cline { 2 - 4 } & Clínicas & Cirúrgicas & Críticas & P \\
\hline $\begin{array}{c}\text { Análise para TEV } \\
\text { Prescrições corretas }\end{array}$ & $85(64,84 \%)$ & $40(51,28 \%)$ & $62(73,81 \%)$ & - \\
Prescrições incorretas & $46(35,11 \%)$ & $38(48,72 \%)$ & $22(26,19 \%)$ & $0,0013^{\mathrm{a}}$ \\
\hline
\end{tabular}

NOTA: ${ }^{a}$ - Teste Qui-Quadrado

Fonte: Autores, 2018

O maior índice de prescrições incorretas para profilaxia de TEV foi encontrado nas unidades cirúrgicas (48,72\%), seguidas pelas unidades clínicas $(35,11 \%)$ e as unidades críticas foram as que menor índice de prescrições incorretas apresentaram $(26,19 \%)$.

Em todas as unidades, predominou a subprescrição como motivo de inadequação da profilaxia, merecendo destaque, mais uma vez, as unidades cirúrgicas, que apresentaram as maiores taxas de subprescrição entre todas as unidades $(94,74 \%, \mathrm{p}<0,001)$ (Tabela 3).

Tabela 3 - Motivos de inadequação das prescrições incorretas por unidade de internamento

\begin{tabular}{cccc}
\hline & \multicolumn{3}{c}{ Motivo de inadequação } \\
\cline { 2 - 4 } & Sobreprescrições & Subprescrições & Posologia incorreta \\
\hline Análise para TEV & & & \\
Unidades clínicas & $16(34,78 \%)$ & $24(52,17 \%)$ & $6(13,04 \%)$ \\
Unidades cirúrgicas & $1(2,63 \%)$ & $36(94,74 \%)$ & $1(2,63 \%)$ \\
Unidades críticas & $2(9,09 \%)$ & $12(54,55 \%)$ & $18(36,36 \%)$ \\
\hline
\end{tabular}

FONTE: Os autores (2018).

Entre os motivos de inadequação das prescrições incorretas para profilaxia de TEV, a subprescrição foi a principal causa de inadequação nos três tipos de unidades estudadas. As unidades cirúrgicas foram as que apresentaram os maiores índices de subprescrição $(94,74 \%)$. As unidades clínicas foram as que apresentaram os maiores índices de 
sobreprescrição (34,78\%). As unidades críticas foram as de maiores índices de prescrições com posologia incorreta $(36,36 \%)$. Valor de $\mathrm{p}<0,001$; teste exato de Fisher.

As unidades clínicas foram as que apresentaram as maiores taxas de sobreprescrição $(34,78 \%, p<0,01)$, e as críticas foram as que apresentaram maior parcela de prescrição com posologia incorreta $(36,36 \%, \mathrm{p}<0,01)$.

\section{DISCUSSÃO}

O estudo demonstrou inadequações das prescrições de profilaxia para tromboembolismo venoso no hospital estudado.

Entre as prescrições analisadas, $36,2 \%$ foram consideradas inadequadas, sendo a subprescrição o principal motivo de inadequação $(67,9 \%, \mathrm{p}=0,013)$. Esse resultado está em concordância com estudos anteriores que avaliaram a qualidade de profilaxias de TEV em outras localidades e que também encontraram a subprescrição como principal motivo de inadequação de suas prescrições ${ }^{11-13}$. Estudo realizado em 4 hospitais de São Paulo, sendo 3 deles universitários, revelou que $29 \%$ dos pacientes sob alto risco para TEV não receberam tromboprofilaxia ${ }^{18}$. Em Vitória - ES, também num hospital de ensino, a taxa de inadequação foi um pouco mais alta, de $47^{(11}$, e num estudo multicêntrico realizado em 21 hospitais de 8 países, essa taxa foi ainda maior, de $60 \% .{ }^{(13)}$ Alguns locais chegaram a registrar níveis de até $77 \%^{12,19}$.

A maior taxa de inadequação foi encontrada nas unidades cirúrgicas $(48,72 \%)$ quando comparada às clínicas $(35,11 \%)$ e às críticas $(26,19 \%)(p=0,013)$. E, entre as prescrições consideradas incorretas para pacientes cirúrgicos, 94,74\% foram devido à subprescrição $(\mathrm{p}<$ $0,01)$. Do mesmo modo, a subprescrição foi a principal desconformidade observada nas unidades críticas $(54,44 \%)$ e também nas clínicas $(52,17 \%)(p<0,01)$. Nessas últimas, a taxa de sobreprescrição foi a maior encontrada entre todos os setores $(34,78 \%, \mathrm{p}<0,01)$.

Essa diferença significativa entre as taxas de inadequação da prescrição da profilaxia para TEV nos diferentes tipos de internamento nem sempre é observada. No estudo de Vitória, 44\% das prescrições cirúrgicas estavam incorretas e, das clínicas, $48 \%$. Nesse mesmo estudo, não houve diferença entre profilaxias para pacientes internados em enfermaria e em UTI ${ }^{11}$. Em contrapartida, na maioria dos estudos em que houve discrepância significativa entre tipos de internamento, pacientes clínicos possuíam maior propensão a não receber a profilaxia adequada em relação aos pacientes cirúrgicos ${ }^{9,20}$. Deheinzelin et al. ${ }^{18}$ demonstraram, nos hospitais de São Paulo, que pacientes cirúrgicos estavam mais propensos a receber tromboprofilaxia adequada em comparação aos pacientes clínicos $(50 \%$ vs. $36 \%, \mathrm{p}<0.05)$, de maneira oposta ao observado pelo presente estudo.

$\mathrm{O}$ menor índice de prescrições inadequadas foi encontrado nas unidades críticas $(26,19 \%, \mathrm{p}=0,013)$. Isso pode ser explicado, em parte, porque esses pacientes apresentavam, em geral, um maior número de fatores de risco para TEV, o que pode ter facilitado a identificação da necessidade da profilaxia ${ }^{21}$. Por outro lado, esses setores foram os que apresentaram maior frequência de posologia inadequada da profilaxia, apesar de correta indicação $(36,36 \%, \mathrm{p}<0,01)$.

Discute-se, com frequência, as razões pelas quais a tromboprofilaxia em pacientes hospitalizados é subutilizada. Algumas das hipóteses postuladas que podem explicar a subprescrição preeminente nesse estudo são: a falta de conscientização dos profissionais médicos sobre o potencial de morbidade relacionada aos eventos tromboembólicos, de modo que os riscos de TEV acabam sendo subestimados ${ }^{22}$; a confusão sobre a profilaxia adequada para diferentes níveis de risco ${ }^{20}$; a falta de familiarização ou concordância dos profissionais médicos com as atuais diretrizes, cuja complexidade pode dificultar a adesão às suas práticas ${ }^{13,22}$; a rotatividade de residentes como fator limitante ao treinamento para uma adequada prática da profilaxia; e, ainda, o não entendimento sobre a quem recai a responsabilidade pela avaliação do risco de TEV de um paciente internado. Nesse sentido, um estudo britânico aponta que alguns médicos inexperientes não sentem que a avaliação do risco de TEV seja de sua responsabilidade ${ }^{23}$.

Outro motivo de grande importância para subutilização da profilaxia é a superestimação do risco de sangramentos com o uso de anticoagulantes profiláticos. É comum o receio da ocorrência de eventos hemorrágicos nesses $\operatorname{casos}^{24}$, já que sangramentos possuem um maior impacto do que eventos trombóticos. Tudo isso leva a um desejo de "não provocar danos" ao paciente, o que culmina com a decisão da omissão da profilaxia ${ }^{21}$. Esse argumento, contudo, não se justifica, uma vez que os benefícios resultantes da prevenção de TEV supera o risco de hemorragias com o uso profilático de anticoagulantes ${ }^{22}$.

Há, ainda, a razão econômica. Existe um propósito de contenção de gastos usado como justificativa para a não prescrição de anticoagulantes para pacientes sob risco de TEV. Esse argumento, contudo, não se sustenta, uma vez que é comprovadamente mais oneroso tratar as complicações decorrentes do TEV do que custear a anticoagulação profilática ${ }^{25}$.

Além disso, muitos estudos têm demonstrado que a implantação de protocolos hospitalares otimiza a segurança e o cuidado dos pacientes internados. O uso do escore de Pádua para estratificação de risco num estudo prospectivo foi associado a uma maior taxa apropriada no uso da tromboprofilaxia ${ }^{15}$. Isso sugere que a falta de um protocolo hospitalar, que poderia facilitar a avaliação da necessidade da prescrição da profilaxia, deve ter contribuído para que as prescrições do hospital estudado estejam em discordância das normas da ACCP. 
Brito GD, et al. Qualidade das prescrições de profilaxia para tromboembolismo venoso em um hospital

A falta de um protocolo pode também ter favorecido os episódios de sobreprescrição, que foram responsáveis por $17,92 \%$ das prescrições incorretas registradas, e também os de erro de posologia $(14,15 \%)$. Supõe-se que essas categorias de inadequação sejam resultado de uma prática médica de prescrição "universal" de anticoagulantes para pacientes hospitalizados, acabando por dispensar uma análise individual do risco e de outras características que influenciariam na escolha da posologia dos fármacos. Essa prática tem como consequência a exposição dos pacientes à anticoagulação desnecessária, a qual sabidamente não é inócua ${ }^{26}$.

Assim, algumas possíveis soluções para reduzir as taxas de inadequação de profilaxia para TEV consistem em investimentos em medidas educacionais aos profissionais médicos, visando o esclarecimento sobre os riscos de TEV no paciente internado, bem como a desconstrução de ideias equivocadas em relação a riscos do uso de anticoagulantes. Também, a ACCP sugere a implementação de protocolos hospitalares, os quais já foram demonstrados como sendo medidas que melhoram o uso da profilaxia e reduzem as taxas de TEV em pacientes internados ${ }^{27,28}$.

Em contrapartida, uma pesquisa realizada no Marrocos demonstrou que intervenções não resultaram em uma melhora significativa da tromboprofilaxia. Inclusive, foi observado um aumento na taxa de sobreprescrição para profilaxia de TEV após a instalação das diretrizes hospitalares ${ }^{24}$.

Esse cenário contraditório aponta para o fato de que a profilaxia para TEV continua sendo um desafio à pratica médica. Por isso, estudos mais direcionados são necessários para melhor compreensão de quais são os pontos críticos que dificultam a adequada prática para profilaxia de TEV em cada localidade. Baseadas nesses resultados é que as intervenções devem ser realizadas.

Uma limitação desse estudo reside no fato de o TCLE ter sido assinado pelos médicos assistentes previamente à coleta de dados, o que pode configurar o chamado efeito Hawthorne, que consiste numa mudança positiva de conduta do profissional ao ter consciência de que se está sendo avaliado ${ }^{29}$. Contudo, acredita-se que esse efeito foi minimizado, uma vez que as prescrições diárias já haviam sido realizadas pelo médico no momento da análise dos prontuários para a pesquisa. $\mathrm{E}$, ainda que essa mudança positiva de conduta tenha acontecido, as taxas de inadequações obtidas foram, mesmo assim, significativas. Isso nos leva a crer que livre do efeito Hawthorne os índices de inadequação poderiam ter sido ainda mais elevados.

Sugerimos, por tudo isso, para estudos futuros, uma mudança da metodologia, a fim de eliminar o efeito Hawthorne. Reiteramos que estudos que aprofundem a caracterização da profilaxia de TEV em populações locais são necessários para uma melhor definição do cenário vigente. Além disso, propomos que essa pesquisa se estenda também para análise de outras profilaxias que são importantes à segurança do paciente internado, como as profilaxias de delirium e de úlcera de pressão.

\section{CONCLUSÃO}

Preocupantes taxas de inadequação das prescrições para profilaxia de tromboembolismo venoso para pacientes internados foram encontradas em um hospital universitário brasileiro, sendo a subprescrição a maior causa da inadequação. Diferentemente da maior parte dos estudos prévios, foi nas unidades cirúrgicas que os maiores índices de profilaxias incorretas foram encontrados nesse estudo. Fundamenta-se, assim, a necessidade de intervenções efetivas, que, baseadas numa análise específica para cada localidade, sejam capazes de adequar as prescrições para profilaxia do tromboembolismo venoso, essa que é uma importante prática de segurança aos pacientes hospitalizados. Quem sabe assim, no futuro, ser internado em qualquer hospital do mundo possa ser seguro.

Agradecimentos: Ao colega Eduardo Augustus Malinowski e à amiga Liane Schneider pelo tempo dedicado ao trabalho.

Participação dos Autores: Giovanna Daneluz de Brito: revisão bibliográfica, coleta de dados, interpretação dos dados, redação do manuscrito (Resumo, Introdução, Discussão e Referências). Guilherme da Silva Fioramonte: revisão bibliográfica, coleta de dados, interpretação dos dados, redação do manuscrito (Abstract, Métodos, Resultados). Gustavo Lenci Marques: idealização do estudo, delineamento da análise, análise estatística, redação do texto (Introdução, Discussão e Conclusão), revisão final do manuscrito.

\section{REFERENCIAS}

1. World Health Organization(WHO). 10 Facts on Patient Safety [cited 2018 Apr 30]. Available from: http://www.who.int/ features/factfiles/patient_safety/en/.

2. Heit JA. Epidemiology of venous thromboembolism. Nat Rev Cardiol. 2015;12(8):464-74. doi: 10.1038/nrcardio.2015.83.

3. Heit JA, Silverstein MD, Mohr DN, Petterson TM, O'Fallon WM, Melton LJ. Risk factors for deep vein thrombosis and pulmonary embolism. Arch Intern Med. 2000;160(6):809. doi: 10.1001/archinte.160.6.809.
4. Anderson FA, Wheeler HB, Goldberg RJ, Hosmer DW, Parwardhan NA, Jovanovic B, et al. A population-based perspective of the hospital incidence and case-fatality rates of deep vein thrombosis and pulmonary embolism: The Worcester DVT study. Arch Intern Med. 1991;151(5):933-8. doi: 10.1001/archinte.1991.00400050081016.

5. Sandler DA, Martin JF. Autopsy proven pulmonary embolism in hospital patients: are we detecting enough deep vein thrombosis? J R Soc Med. 1989;82(4):203-5. doi: $10.1177 / 014107688908200407$.

6. Amin NA, Lin J, Johnson BH, Schulman KL. Clinical 
and economic outcomes with appropriate or partial prophylaxis. Thromb Res. 2010;125(6):513-25. doi: 10.1016/j.thromres.2009.10.018.

7. Cohoon KP, Leibson CL, Ransom JE, Ashrani AA, Petterson $\mathrm{TM}$, Long KH, et al. Costs of venous thromboembolism associated with hospitalization for medical ilness. Am J Manag Care. 2015;21(4):e255-e263. Available from: https:// www.ajmc.com/journals/issue/2015/2015-vol21-n4/costs-ofvenous-thromboembolism-associated-with-hospitalizationfor-medical-illness.

8. Cohen AT, Davidson BL, Gallus AS, Lassen MR, Prins MH, Tomkowski W, et al. Efficacy and safety of fondaparinux for the prevention of venous thromboembolism in older acute medical patients: randomised placebo controlled trial. BMJ. 2006;332:325. doi:10.1136/bmj.38733.466748.7C.

9. Dentali F, Douketis JD, Gianni M, Lim W, Crowther MA. Meta-analysis : anticoagulant prophylaxis to prevent symptomatic venous thromboembolism in hospitalized medical patients. Ann Intern Med. 2007;146:277-88. doi: 10.7326/0003-4819-146-4-200702200-00007.

10. Shekelle PG, Wachter RM, Pronovost PJ, et al. Making Health Care Safer II: an updated critical analysis of the evidence for patient safety practices. Rockville (MD): Agency for Healthcare Research and Quality (US); 2013. (Evidence Reports/Technology Assessments, no. 211). Available from: https://www.ncbi.nlm.nih.gov/books/NBK133369/.

11. Carneiro JLDA, Targueta GP, Marino LO. Avaliação da profilaxia do tromboembolismo venoso em hospital de grande porte. Rev Col Bras Cir. 2010;37(3):204-10. http://dx.doi. org/10.1590/S0100-69912010000300008.

12. Pitta GB, Leite TL, Silva MD, Melo CF, Calheiros GD, et al. Evaluation of the use of prophylaxis for deep venous thrombosis in a teaching hospital. J Vasc Bras. 2007;6(4):34451. http://www.scielo.br/pdf/jvb/v6n4/en_v6n4a08.pdf.

13. Tapson VF, Decousus H, Pini M, Chong BH, Froehlich JB, Monreal M, et al. Venous thromboembolism prophylaxis in acutely ill hospitalized medical patients: findings from the international medical prevention registry on venous thromboembolism. Chest. 2007;132(3):936-45. doi: 10.1378/ chest.06-2993.

14. Lester W, Freemantle N, Begaj I, Ray D, Wood J, Pagano D. Fatal venous thromboembolism associated with hospital admission: A cohort study to assess the impact of a national risk assessment target. Heart. 2013;99(23):1734-9. doi: 10.1136/heartjnl-2013-304479.

15. Germini F, Agnelli G, Fedele M, Galli MG, Giustozzi M, Marcucci M, et al. Padua prediction score or clinical judgment for decision making on antithrombotic prophylaxis: a quasi-randomized controlled trial. J Thromb Thrombolysis. 2016;42(3):336-9. doi: 10.1007/s11239-016-1358-z.

16. Guyatt GH, Akl EA, Crowther M, Gutterman DD, Schuünemann HJ; American College of Chest Physicians Antithrombotic Therapy and Prevention of Thrombosis Panel. Executive summary: Antithrombotic therapy and prevention of thrombosis. 9th ed. American College of Chest Physicians evidence-based clinical practice guidelines. Chest.
2012;141(2 Supp1.):7-47. doi: 10.1378/chest.1412S3.

17. R Core Team. R: A Language and Environment for Statistical Computing [Internet]. Vienna, Austria; 2016. Available from: https://www.R-project.org/

18. Deheinzelin D, Braga AL, Martins LC, Martins MA, Hernandez A, Yoshida WB, et al. Incorrect use of thromboprophylaxis for venous thromboembolism in medical and surgical patients: results of a multicentric, observational and cross-sectional study in Brazil. J Thromb Haemost. 2006;4(6):1266-70. doi: 10.1111/j.1538-7836.2006.01981.x.

19. Pitta GBB, Gomes RR. A frequência da utilização de profilaxia para trombose venosa profunda em pacientes clínicos hospitalizados. J Vasc Bras. 2010;9(4):220-8. http://dx.doi. org/10.1590/S1677-54492010000400003.

20. Goldhaber SZ, Tapson VF. DVT FREE Steering Comittee. A prospective registry of 5,451 patients with ultrasound-confirmed deep vein thrombosis. Am J Cardiol. 2004;93(2):259-62. doi: 10.1016/j.amjcard.2003.09.057.

21. Jelic S. Direct characterization of the vascular endothelium in sleep apnea. Chest. 2011;140(6):1400-01. doi: 10.1378/ chest.11-1376.

22. Arnold DM, Kahn SR, Shrier I. Missed Opportunities for Prevention of Venous Thromboembolism: an evaluation of the use of thromboprophylaxis. Chest. 2001;120:1964-71. doi: 10.1378/chest.120.6.1964.

23. McFarland L, Murray E, Harrison S, Heneghan C, Ward A, Fitzmaurice D, et al. Current practice of venous thromboembolism prevention in acute trusts: a qualitative study. BMJ Open. 2014;4e005074. doi: 10.1136/ bmjopen-2014-005074.

24. Mezalek ZT, Nejjari C, Essadouni L, Serraj M, Ammouri W, Belkhadir NKZ et al. Evaluation and management of thromboprophylaxis in Moroccan hospitals at national level: the Avail-MoNa study. J Thromb Thrombolysis. 2018;46(1):113-9. doi: 10.1007/s11239-018-1657-7.

25. Bergqvist D, Lindgren B, Matzsch T. Comparison of the cost of preventing postoperative deep vein thrombosis with either unfractionated or low molecular weight heparin. Br J Surg. 1996;83(11):1548-52. doi: 10.1002/bjs. 1800831117.

26. Harrington R, Ansell J. Risk-benefit assessment of anticoagulant therapy. Drug Saf. 1991;6(I):54-69. doi: 10.2165/00002018-199106010-00006.

27. Kucher. N, Koo. S, Quiroz. R, Cooper JM, Paterno MD, Soukonnikov B, et al. Eletronic alerts to prevent venous thromboembolism among hospitalized patients. N Eng J Med. 2010;363(1):1-3. doi: 10.1056/NEJMoa041533.

28. Hirsh J, Guyatt G, Alberts GW, Schünemann HJ. The Seventh ACCP Conference on Antithrombotic and Thrombolytic Therapy: Evidence-Based Guidelines. Chest. 2004;126(3 Suppl):172-3. doi: 10.1378/chest.126.3_suppl.172S.

29. Wickstrom G, Bendix T. The "Hawthorne effect" - what did the original Hawthorne studies actually show? Scand J Work Environ Health. 2000;26(4):363-7. doi:10.5271/sjweh.555.

Recebido: 30.06 .19

Aceito: 21.10 .19 